University of Wollongong

Research Online

Faculty of Engineering and Information

Faculty of Engineering and Information

Sciences - Papers: Part A

Sciences

$1-1-2012$

\title{
Robust sampled-data control of structures subject to parameter uncertainties and actuator saturation
}

\author{
Haiping Du \\ University of Wollongong, hdu@uow.edu.au \\ Nong Zhang \\ University Of Technology Sydney \\ Bijan Samali \\ University Of Technology Sydney \\ Fazel Naghdy \\ University of Wollongong, fazel@uow.edu.au
}

Follow this and additional works at: https://ro.uow.edu.au/eispapers

Part of the Engineering Commons, and the Science and Technology Studies Commons

Research Online is the open access institutional repository for the University of Wollongong. For further information contact the UOW Library: research-pubs@uow.edu.au 


\title{
Robust sampled-data control of structures subject to parameter uncertainties and actuator saturation
}

\begin{abstract}
"This paper presents a robust sampled-data controller design approach for vibration attenuation of civil structures considering parameter uncertainties and actuator saturation. The parameter uncertainties belong to polytopic form and are assumed to be the variations of the structural stiffness and damping. Regarding the uncertain sampling problem encountered in real world applications, the sampling period designed for the controller is allowed to be variable within a given bound. In order to obtain reduced peak response quantities, the energy-to-peak performance used to describe the peak values of the control output under all possible energy-bounded disturbances is optimised. The robust sampled-data state feedback controller is obtained in terms of the solvability of certain linear matrix inequalities (LMIs). The applicability of the proposed approach is demonstrated by a numerical example on vibration control of a building structure subject to seismic excitation. It is validated by the simulation results confirming that the designed controllers can effectively attenuate the structural vibration and keep the system stability while there are parameter uncertainties and actuator saturation constraints. (C) 2011 Elsevier Ltd. All rights reserved."
\end{abstract}

\section{Keywords}

uncertainties, saturation, robust, sampled, data, control, actuator, structures, subject, parameter

\section{Disciplines}

Engineering | Science and Technology Studies

\section{Publication Details}

Du, H., Zhang, N., Samali, B. \& Naghdy, F. (2012). Robust sampled-data control of structures subject to parameter uncertainties and actuator saturation. Engineering Structures, 36 39-48. 


\title{
Robust Sampled-data Control of Structures Subject to
}

\section{Parameter Uncertainties and Actuator Saturation}

\author{
Haiping Du * Nong Zhang ${ }^{\dagger} \quad$ Bijan Samali ${ }^{\ddagger} \quad$ Fazel Naghdy ${ }^{\S}$
}

October 26, 2011

\begin{abstract}
This paper presents a robust sampled-data controller design approach for vibration attenuation of civil structures considering parameter uncertainties and actuator saturation. The parameter uncertainties belong to polytopic form and are assumed to be the variations of the structural stiffness and damping. Regarding the uncertain sampling problem encountered in real world applications, the sampling period designed for the controller is allowed to be variable within a given bound. In order to obtain reduced peak response quantities, the energy-to-peak performance used to describe the peak values of the control output under all possible energy-bounded disturbances is optimised. The robust sampled-data state feedback controller is obtained in terms of the solvability of certain linear matrix inequalities (LMIs). The applicability of the proposed approach is demonstrated by a numerical example on vibration control of a building structure subject to seismic excitation. It is validated by the simulation results confirming that the designed controllers can effectively attenuate the structural vibration and keep the system stability while there are parameter uncertainties and actuator saturation constraints.
\end{abstract}

${ }^{*}$ School of Electrical, Computer and Telecommunications Engineering, University of Wollongong, Wollongong, NSW 2522, Australia. Email: hdu@uow.edu.au.

${ }^{\dagger}$ Mechatronics and Intelligent Systems, Faculty of Engineering, University of Technology, Sydney, Australia.

${ }^{\ddagger}$ Infrastructure and the Environment, Faculty of Engineering, University of Technology, Sydney, Australia.

${ }_{\S}^{\S}$ School of Electrical, Computer and Telecommunications Engineering, University of Wollongong, Australia. 
Keywords: Structural vibration control; Sampling rate; Parameter uncertainties; Actuator saturation.

\section{Introduction}

With the rapid development of computer technology, digital controllers are becoming a reality in many engineering applications, in which a digital computer is used to sample and quantify a continuous-time measurement signal and produce a discrete-time control input signal which is further converted back into a continuous-time control input signal using a zero-order hold. Since physical plants are continuous-time systems in real world, the control systems that use digital controllers involve both continuous-time and discrete-time signals in the continuous-time frame and are referred to as sampled-data systems.

Analysis and synthesis of sampled-data systems have been investigated in a number of papers (see for example $[1,2,3,4,5])$. In civil engineering, the control of building structures subject to earthquakes or strong winds has received considerable attention over the past three decades and much effort has been devoted to the development of control devices and algorithms $[6,7,8]$. With recent focus on wireless monitoring and control of structural systems $[9,10,11,12]$ based on networked control technique [13], studying sampleddata control problem for structures is becoming significant. Classical solutions to this type of feedback control problem as well as their applications in civil engineering can be found in the literature $[14,15,16]$, where optimal discrete-time and sampled-data control algorithms taking into account external excitations were developed for structural engineering applications. Some practical issues such as the effect of sampling frequency, time delay and actuator dynamics were addressed. The methods were numerically validated on the building examples. However, although the effect of sampling frequency was studied in those research and it was shown that the control efficiency were improved significantly with higher sampling frequency, it is noted that the controller design given in those studies is fully dependent on a given sampling rate. That means the controller design is fully based on the assumption that the sampling is made periodic and the controller should be re-designed once the sampling frequency is changed anyway. In practice, the sampling 
frequency can be varied in terms of the digital realisation requirement and the uncertain sampling may happen when the sampler contains uncertainties or the mathematical model used is not ideally consistent with the sampling equipment. Therefore, designing a sampled-data controller that is robust to the variable sampling rate is necessary.

The parameter uncertainties are one of the most critical issues to a control system as they can affect both the performance and the stability of the control system. Parameter uncertainties may come from modelling errors, variations in material properties, and changing load environments which make the system description for the structural models inevitably containing uncertainties of different nature and level $[6,17,18]$. On the other hand, any actuation mechanisms are subject to inherent physical limitations. The saturation on actuator capacity takes on added importance in structural applications, and in earthquake design in particular [19]. For structures, robust continuous-time controller design considering practical issues like parameter uncertainties, actuator saturation, actuator failure, time delay, etc., was recently studied by, for example, [20, 21, 22, 23, 24]. As indicated in the concluding remarks of [16], considering actuator saturation and model uncertainties in the sampled-data controller design process should be a logical next step. This motivates the present study.

This paper concerns with the robust sampled-data controller design for buildings with parameter uncertainties and actuator saturation constraint. The objective is to design a state feedback controller such that the closed-loop system is asymptotically stable with an optimal disturbance attenuation subject to parameter uncertainties and actuator saturation. The parameter uncertainties dealt with are of a polytopic type, the sampling rate is designed to be variable, and the energy-to-peak performance [25] is used to obtain good peak response quantities. Based on the recently developed input delay approach $[4,5]$, sufficient conditions for designing such a controller are derived in terms of linear matrix inequalities (LMIs) which can be resolved efficiently using the available software Matlab LMI Toolbox. To validate the effectiveness of the approach, the designed controllers are applied to reduce the vibration of a seismic-excited building structure. Simulation results show good vibration attenuation performance and system robust stability in spite of parameter uncertainties, actuator saturation, and variable sampling rate. 
The rest of this paper is organised as follows. Section 2 presents the problem description for sampleddata control of uncertain structures. Section 3 derives the conditions for designing the robust controller. Section 4 provides an application example to validate the effectiveness of the approach developed. Finally, we conclude our findings in Section 5.

Notation: $\mathbb{R}^{n}$ denotes the $n$-dimensional Euclidean space and $\mathbb{R}^{n \times m}$ the set of all $n \times m$ real matrices. For a real symmetric matrix $W$, the notation of $W \succ 0(W \prec 0)$ is used to denote its positive- (negative-) definiteness. $\mathbf{I}$ is used to denote the identity matrix of appropriate dimension. When a matrix is equal to $\mathbf{0}$, in such case, $\mathbf{0}$ is used to denote the zero matrix of appropriate dimension. To simplify notation, $*$ is used to represent a block matrix which is readily inferred by symmetry.

\section{Sampled-data Control of Uncertain Structure}

Consider an $n$ degree-of-freedom (DOF) actively controlled building structure subject to external excitations, the governing equation is written as

$$
M \ddot{x}(t)+C \dot{x}(t)+K x(t)=E w(t)+H u(t),
$$

where $x(t)=\left[x_{1}(t), x_{2}(t), \ldots, x_{n}(t)\right]^{T}$, and $x_{n}(t)$ is the $n$th floor relative displacement with respect to ground; $\dot{x}(t)$ and $\ddot{x}(t)$ are the first and second time derivatives of $x(t)$, respectively; $u(t)=\left[u_{1}(t), u_{2}(t), \ldots, u_{r}(t)\right]^{T}$, $u_{r}(t)$ is the $r$ th control force; $H \in \mathbb{R}^{n \times r}$ gives the location of the $r$ controllers; $w(t)$ is the external excitation; $E$ is a vector denoting the influence of external excitation; $M, C, K \in \mathbb{R}^{n \times n}$ are the mass, damping, and stiffness matrices of the structure, respectively.

Define the state vector as $q(t)=\left[\begin{array}{ll}x^{T}(t) & \dot{x}^{T}(t)\end{array}\right]^{T}$, the state space representation of the structure in (1) can be expressed as

$$
\dot{q}(t)=A q(t)+B_{w} w(t)+B u(t)
$$

where

$$
A=\left[\begin{array}{cc}
0 & I \\
-M^{-1} K & -M^{-1} C
\end{array}\right], \quad B_{w}=\left[\begin{array}{c}
0 \\
M^{-1} E
\end{array}\right], \quad B=\left[\begin{array}{c}
0 \\
M^{-1} H
\end{array}\right] .
$$


Consider system (2) has parameter uncertainties, and in particular, the parameter uncertainties are induced by the variations of stiffnesses and damping coefficients, the parameter uncertainties in matrices of system (2) can belong to a polytopic set described by $\kappa$ vertices, then, the system matrix $A$ can be expressed as

$$
A(\xi) \in \Theta \triangleq\left\{A(\xi) \mid A(\xi)=\sum_{i=1}^{\kappa} \xi_{i} A_{i} ; \xi_{i} \geqslant 0 ; \sum_{i=1}^{\kappa} \xi_{i}=1\right\}
$$

where $\xi$ is used to characterise the parameter uncertainty and is assumed to be varied in a polytope of vertices $\xi_{1}, \xi_{2}, \ldots, \xi_{\kappa}$, i.e., $\xi \in \Theta \triangleq C o\left\{\xi_{1}, \xi_{2}, \ldots, \xi_{\kappa}\right\}$, where the symbol $C o$ denotes the convex hull and $\Theta$ denotes a given convex bounded polyhedral domain.

With further consideration on the actuator saturation, system (2) is expressed as

$$
\dot{q}(t)=A(\xi) q(t)+B_{w} w(t)+B \cdot \operatorname{sat}(u(t))
$$

where the actuator saturation expression $\operatorname{sat}(u)$ is in the decentralised saturation form, that is, $[\operatorname{sat}(u)]_{i}=\operatorname{sat}\left(u_{i}\right)$, where $i=1,2, \ldots, r$, and $\operatorname{sat}\left(u_{i}\right)$ is the standard saturation function with the limit of $u_{\lim _{i}}$ for the $i$ th actuator, that is,

$$
\operatorname{sat}\left(u_{i}\right)= \begin{cases}u_{i}, & \left|u_{i}\right| \leqslant u_{\lim _{i}} \\ \operatorname{sign}\left(u_{i}\right) u_{\lim _{i}}, & \left|u_{i}\right|>u_{\lim _{i}}\end{cases}
$$

Using the following transform $[26,27,28]$

$$
\operatorname{sat}(u)=\Psi(\eta) u
$$

where $\Psi(\eta)=\operatorname{diag}\left\{\eta_{1}, \ldots, \eta_{i}, \ldots, \eta_{r}\right\}, \eta_{i} \triangleq \frac{\operatorname{sat}\left(u_{i}\right)}{u_{i}}$ with $\eta_{i}=1$ if $u_{i}=0$, equation (4) can now be written as

$$
\dot{q}(t)=A(\xi) q(t)+B_{w} w(t)+B \Psi(\eta) u(t) .
$$

To obtain a high gain controller as that in [26], the command to the $i$ th actuator is allowed to be $\delta_{i} u_{\lim _{i}}$ for an arbitrary scalar $\delta_{i}>1$. Therefore, the resulting $\eta_{i}$ will be bounded by 1 and $1 / \delta_{i}$, that is,

$$
\eta \in \mathcal{P} \triangleq\left\{\eta: \frac{1}{\delta_{i}} \leqslant \eta_{i} \leqslant 1, i=1,2, \ldots, r\right\}
$$


Accordingly, the vertex set associated with (8) is denoted as

$$
\mathcal{P}_{\text {vex }} \triangleq\left\{\eta: \eta_{i}=\frac{1}{\delta_{i}} \text { or } \eta_{i}=1, i=1,2, \ldots, r\right\}
$$

and $\Psi(\eta)$ can be expressed as $\Psi(\eta)=\sum_{i=1}^{2 r} \zeta_{i} \Psi\left(\eta_{i}\right)=\sum_{i=1}^{2 r} \zeta_{i} \Psi_{i}$, where $\zeta_{i} \geqslant 0$ and $\sum_{i=1}^{2 r} \zeta_{i}=1$.

In this paper, the external excitation signal $w(t)$ is assumed to be bounded and with finite energy, that is,

$$
\|w\|_{2} \triangleq \sqrt{\int_{0}^{\infty} w^{T}(t) w(t) d t}<\infty
$$

i.e., $w(t) \in L_{2}[0, \infty)$. This is one possible specification for a class of design loads that the engineering structures are designed to resist, for example, a class of design earthquakes whose intensity and associated total energy is specified on a Richter scale [25].

To design a controller for active vibration attenuation of structures under external excitations, the control output should be defined so that the performance index from the external excitation to the control output can be realised with the specified requirement. For system (7), we define the control output as

$$
z(t)=C_{z} q(t)
$$

where $C_{z}$ is a constant matrix which defines the interested output variables.

Now, it is assumed that the state variables of the building structure are measured at time instants $0=t_{0}<t_{1}<\cdots<t_{k}<t_{k+1}<\cdots$, and only $q\left(t_{k}\right)$ are available for interval $t_{k} \leqslant t<t_{k+1}$. Then, for the uncertain system (7), we are interested in designing a state feedback controller in the form of

$$
u(t)=u\left(t_{k}\right)=K_{c} q\left(t_{k}\right), t_{k} \leqslant t<t_{k+1}
$$

where $K_{c} \in \mathbb{R}^{r \times n}$ is the state feedback gain matrix to be designed. With the controller (12), the closed-loop system is becoming

$$
\dot{q}(t)=A(\xi) q(t)+B_{w} w(t)+B \Psi(\eta) K_{c} q\left(t_{k}\right), t_{k} \leqslant t<t_{k+1}
$$


In order to achieve good peak response qualities, the paper aims to design a controller (12) such that the closed-loop system (13) is asymptotically stable for all admissible parameter uncertainties in spite of the saturation constraint, and the closed-loop system (13) guarantees, under zero initial condition, $\|z\|_{\infty}<$ $\gamma\|w\|_{2}$, i.e., energy-to-peak performance, where $\gamma>0$ is a prescribed constant, for all non-zero $w \in L_{2}[0, \infty)$.

The block diagram for the sampled-data control of building structure is shown in Figure 1. It is assumed in this study that the sampling of the measurement is synchronised with the holding of the control signal, and the interval between any two sampling instants is bounded by

$$
t_{k+1}-t_{k} \leqslant h, \forall k \geqslant 0
$$

where $h>0$ is the maximum sampling interval, i.e., the maximum sampling interval is bounded. It does not require the sampling to be periodic, and the designed controller (12) should be effective for any sampling frequency higher than $1 / h$.

\section{Robust Sampled-data Controller Design}

It is noticed that the sampling instant $t_{k}$ can be represented as

$$
t_{k}=t-\left(t-t_{k}\right)=t-\tau(t)
$$

where $\tau(t)=t-t_{k}$. Then, we obtain

$$
u(t)=u\left(t_{k}\right)=u(t-\tau(t)), t_{k} \leqslant t<t_{k+1}
$$

where $u\left(t_{k}\right)$ is a discrete-time control signal and the time-varying delay $\tau(t)=t-t_{k} \leqslant h$ is piecewise-linear with derivative $\dot{\tau}(t)=1$ for $t \neq t_{k}$. Using (16), the closed-loop system (13) is rewritten as

$$
\dot{q}(t)=A(\xi) q(t)+B_{w} w(t)+B \Psi(\eta) K_{c} q(t-\tau(t)),
$$

which is a continuous-time system with an uncertain and bounded delay $\tau(t)$ in state. Note that the sampleddata system (13) can be seen as a particular class of the state-delayed system (17). The asymptotic stability of system (13) is guaranteed if system (17) is asymptotically stable. 
Now, choose a Lyapunov-Krasovskii functional candidate for system (17) as

$$
V(t)=q^{T}(t) P q(t)+\int_{-h}^{0} \int_{t+\beta}^{t} \dot{q}^{T}(\alpha) Q \dot{q}(\alpha) d \alpha d \beta
$$

where $P=P^{T}, P \succ 0, Q=Q^{T}, Q \succ 0$. Then, the time derivative of $V(t)$ along the solution of system (17) gives

$$
\begin{aligned}
\dot{V}(t) & =\dot{q}^{T}(t) P q(t)+q^{T}(t) P \dot{q}(t)+h \dot{q}^{T}(t) Q \dot{q}(t)-\int_{t-h}^{t} \dot{q}^{T}(\alpha) Q \dot{q}(\alpha) d \alpha \\
& \leqslant \dot{q}^{T}(t) P q(t)+q^{T}(t) P \dot{q}(t)+h \dot{q}^{T}(t) Q \dot{q}(t)-\int_{t-\tau(t)}^{t} \dot{q}^{T}(\alpha) Q \dot{q}(\alpha) d \alpha \\
& =\frac{1}{\tau(t)} \int_{t-\tau(t)}^{t} \Sigma(t, \alpha) d \alpha,
\end{aligned}
$$

where

$$
\begin{aligned}
\Sigma(t, \alpha)= & \dot{q}^{T}(t) P q(t)+q^{T}(t) P \dot{q}(t)+h \dot{q}^{T}(t) Q \dot{q}(t)-\tau(t) \dot{q}^{T}(\alpha) Q \dot{q}(\alpha) \\
= & 2 q^{T}(t) P\left(A(\xi) q(t)+B_{w} w(t)+B \Psi(\eta) K_{c} q(t-\tau(t))\right. \\
& +\left(A(\xi) q(t)+B_{w} w(t)+B \Psi(\eta) K_{c} q(t-\tau(t))^{T} h Q\left(A(\xi) q(t)+B_{w} w(t)+B \Psi(\eta) K_{c} q(t-\tau(t))\right.\right. \\
& -\tau(t) \dot{q}^{T}(\alpha) Q \dot{q}(\alpha) .
\end{aligned}
$$

By the Newton-Leibniz formula, we have

$$
\int_{t-\tau(t)}^{t} \dot{q}(\alpha) d \alpha=q(t)-q(t-\tau(t))
$$

Then, for any appropriately dimensioned matrices

$$
X(\xi)=\sum_{i=1}^{\kappa} \xi_{i} X_{i}, Y(\xi)=\sum_{i=1}^{\kappa} \xi_{i} Y_{i}
$$

and we have

$$
\Lambda=\frac{1}{\tau(t)} \int_{t-\tau(t)}^{t}\left[q^{T}(t) q^{T}(t-\tau(t))\right]\left[\begin{array}{c}
X(\xi) \\
Y(\xi)
\end{array}\right][q(t)-q(t-\tau(t))-\tau(t) \dot{q}(\alpha)] d \alpha=0
$$

Adding $2 \Lambda$ to the right hand of (19), we have

$$
\dot{V}(t)-w^{T}(t) w(t) \leqslant \frac{1}{\tau(t)} \int_{t-\tau(t)}^{t} \vartheta^{T}(t, \alpha) \Pi \vartheta(t, \alpha) d \alpha,
$$




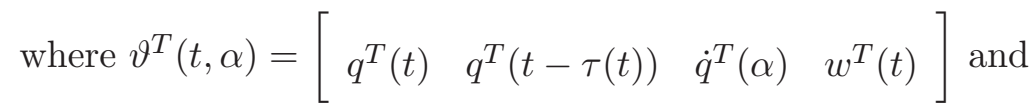

$$
\Pi=\left[\begin{array}{cccc}
\Pi_{11} & \Pi_{12} & -\tau(t) X(\xi) & P B_{w}+h A^{T}(\xi) Q B_{w} \\
* & \Pi_{22} & -\tau(t) Y(\xi) & h K_{c}^{T} \Psi^{T}(\eta) B^{T} Q B_{w} \\
* & * & -\tau(t) Q & \mathbf{0} \\
* & * & * & h B_{w}^{T} Q B_{w}-\mathbf{I}
\end{array}\right],
$$

where $\Pi_{11}=P A(\xi)+A^{T}(\xi) P+h A^{T}(\xi) Q A(\xi)+X(\xi)+X^{T}(\xi), \Pi_{12}=P B \Psi(\eta) K_{c}+h A^{T}(\xi) Q B \Psi(\eta) K_{c}-$ $X(\xi)+Y^{T}(\xi)$, and $\Pi_{22}=h K_{c}^{T} \Psi^{T}(\eta) B^{T} Q B \Psi(\eta) K_{c}-Y^{T}(\xi)-Y(\xi)$.

When assuming the zero-disturbance input, i.e., $w(t) \equiv 0$, if $\Pi \prec 0$, then from $(23), \dot{V}(t)<0$ is established and the asymptotic stability of the closed-loop system (17) and (13) is guaranteed.

Assume zero initial condition, i.e., $q(t)=\phi(t)=0, \forall t \in[-h, 0]$, then, we have $\left.V(t)\right|_{t=0}=0$. And for any non-zero disturbance $w \in L_{2}[0, \infty)$ and $t \geqslant 0$, if $\Pi \prec 0$, there holds,

$$
V(t)-\left.V(t)\right|_{t=0}-\int_{0}^{t} w^{T}(s) w(s) d s<0
$$

and $V(t)<\int_{0}^{t} w^{T}(s) w(s) d s$.

By Schur complement, $\Pi \prec 0$ is equivalent to

$$
\left[\begin{array}{rrrrr}
P A(\xi)+A^{T}(\xi) P+X(\xi)+X^{T}(\xi) & P B \Psi(\eta) K_{c}-X(\xi)+Y^{T}(\xi) & -X(\xi) & P B_{w} & A^{T}(\xi) \\
* & -Y^{T}(\xi)-Y(\xi) & -Y(\xi) & \mathbf{0} & K_{c}^{T} \Psi^{T}(\eta) B^{T} \\
* & * & -\tau(t)^{-1} Q & \mathbf{0} & \mathbf{0} \\
* & * & * & -\mathbf{I} & B_{w}^{T} \\
* & * & * & * & -h^{-1} Q^{-1}
\end{array}\right] \prec
$$

Define $L \triangleq P^{-1}$, and pre- and post-multiplying (26) by $\operatorname{diag}\left(\begin{array}{ccccc}L & L & L & I & I\end{array}\right)^{T}$ and its transpose, re- 
spectively, we obtain

$$
\left[\begin{array}{ccccc}
\Xi & B \Psi(\eta) K_{c} L-L X(\xi) L+L Y^{T}(\xi) L & -L X(\xi) L & B_{w} & L A^{T}(\xi) \\
* & -L Y^{T}(\xi) L-L Y(\xi) L & -L Y(\xi) L & \mathbf{0} & L K_{c}^{T} \Psi^{T}(\eta) B^{T} \\
* & * & -\tau^{-1}(t) L Q L & \mathbf{0} & \mathbf{0} \\
* & * & * & -\mathbf{I} & B_{w}^{T} \\
* & * & * & * & -h^{-1} Q^{-1}
\end{array}\right] \prec 0
$$

where $\Xi=A(\xi) L+L A^{T}(\xi)+L X(\xi) L+L X^{T}(\xi) L$. By defining $\bar{X}(\xi)=L X(\xi) L, \bar{Y}(\xi)=L Y(\xi) L, \bar{K}_{c}=K_{c} L$, and $R=Q^{-1}$ in (27), we obtain

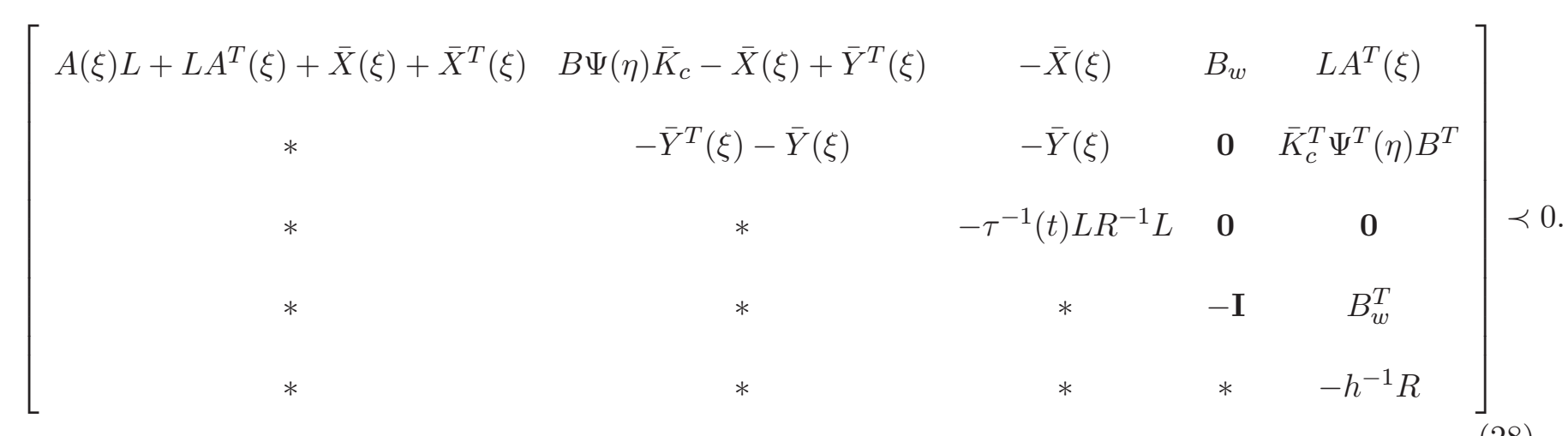

It is noticed that $(R-L) R^{-1}(R-L) \succeq 0$ since $R \succ 0$, which is equivalent to

$$
L R^{-1} L \preceq R-2 L .
$$

Therefore, from $(29)$ and $\tau(t) \leqslant h$, if

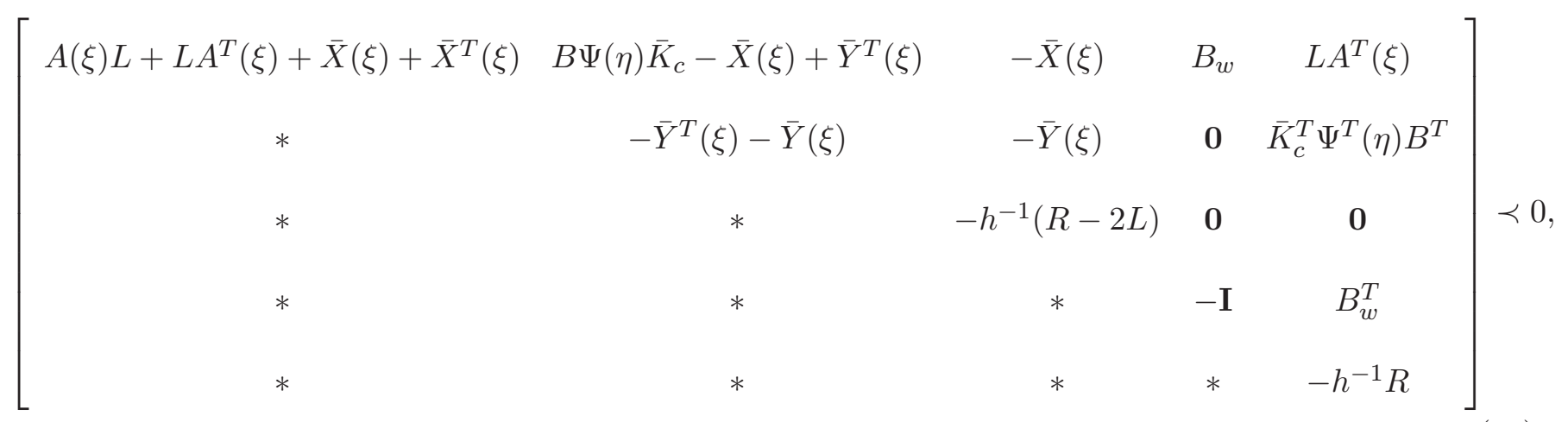

then, the inequality (28) can be established. Substituting $A(\xi)=\sum_{i=1}^{\kappa} \xi_{i} A_{i}, \bar{X}(\xi)=\sum_{i=1}^{\kappa} \xi_{i} \bar{X}_{i}, \bar{Y}(\xi)=\sum_{i=1}^{\kappa} \xi_{i} \bar{Y}_{i}$, 
and $\Psi(\eta)=\sum_{j=1}^{2 r} \zeta_{j} \Psi_{j}$ into (30), we readily obtain the equivalent condition as

$$
\left[\begin{array}{ccccc}
A_{i} L+L A_{i}^{T}+\bar{X}_{i}+\bar{X}_{i}^{T} & B \Psi_{j} \bar{K}_{c}-\bar{X}_{i}+\bar{Y}_{i}^{T} & -\bar{X}_{i} & B_{w} & L A_{i}^{T} \\
* & -\bar{Y}_{i}^{T}-\bar{Y}_{i} & -\bar{Y}_{i} & \mathbf{0} & \bar{K}_{c}^{T} \Psi_{j}^{T} B^{T} \\
* & * & -h^{-1}(R-2 L) & \mathbf{0} & \mathbf{0} \\
* & * & * & -\mathbf{I} & B_{w}^{T} \\
* & * & * & * & -h^{-1} R
\end{array}\right] \prec 0, i \in[1, \kappa], j \in[1,2 r] .
$$

Furthermore, using Schur complement, the feasibility of the following inequality

$$
\left[\begin{array}{cc}
P & C_{z}^{T} \\
C_{z} & \gamma^{2} \mathbf{I}
\end{array}\right] \succ 0
$$

guarantees $C_{z}^{T} C_{z} \prec \gamma^{2} P$. At the same time, it can be derived from (25) that $q^{T}(t) P q(t)<\gamma^{2} \int_{0}^{t} w^{T}(s) w(s) d s$ if $\Pi \prec 0$ is guaranteed. Then, it can easily be established from (11) that for all $t \geqslant 0$,

$$
z^{T}(t) z(t)=q^{T}(t) C_{z}^{T} C_{z} q(t)<\gamma^{2} q^{T}(t) P q(t)<\gamma^{2} \int_{0}^{t} w^{T}(s) w(s) d s \leqslant \gamma^{2} \int_{0}^{\infty} w^{T}(s) w(s) d s .
$$

Taking the supremum over $t \geqslant 0$ yields $\|z\|_{\infty}<\gamma_{2}\|w\|_{2}$ for all $w \in L_{2}[0, \infty)$, that is, the energy-to-peak performance is established. Similarly, condition (32) is transformed to

$$
\left[\begin{array}{cc}
L & L C_{z}^{T} \\
C_{z} L & \gamma^{2} \mathbf{I}
\end{array}\right] \succ 0 .
$$

On the other hand, from (12), the constraint $\left|u_{i}\right| \leqslant \delta_{i} u_{\lim i}$ can be expressed as

$$
\left|K_{c i} q(t)\right| \leqslant \delta_{i} u_{\lim i}
$$

where $K_{c i}$ is the $i$ th row of $K_{c}$. Let $\Omega\left(K_{c}\right)=\left\{q(t)|| q^{T}(t) K_{c i}^{T} K_{c i} q(t) \mid \leqslant\left(\delta_{i} u_{\lim i}\right)^{2}\right\}$, the equivalent condition for an ellipsoid $\Omega(P, \rho)=\left\{q(t) \mid q^{T}(t) P q(t) \leqslant \rho\right\}$ being a subset of $\Omega\left(K_{c}\right)$ is [29]

$$
K_{c i}\left(\frac{P}{\rho}\right)^{-1} K_{c i}^{T} \leqslant\left(\delta_{i} u_{\lim i}\right)^{2} .
$$

By Schur complement, inequality (36) can be written as

$$
\left[\begin{array}{cc}
\left(\delta_{i} u_{\lim i}\right)^{2} \mathbf{I} & K_{c_{i}} \\
K_{c_{i}}^{T} & \frac{P}{\rho}
\end{array}\right] \succeq 0 .
$$


Using the definitions $L=P^{-1}$ and $\bar{K}_{c}=K_{c} P^{-1}$, inequality (37) is equivalent to

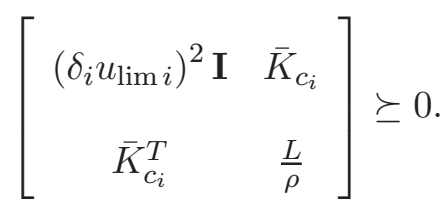

We now summarise the controller design procedure in the following theorem. The proof of this theorem is evident from the above presented analysis, and will be omitted for brevity.

Theorem 1 Consider the building system (1), there exists a state feedback controller in the form of (12) such that the closed-loop system in (17) is asymptotically stable with guaranteed $\left\|z_{2}\right\|_{\infty}<\gamma\|w\|_{2}$ under parameter uncertainties and actuator saturation constraint defined in (3) and (5), if for given scalars $\rho>0$, $\gamma>0, \delta>0$, and $h>0$, there exist matrices $L \succ 0, R \succ 0, \bar{X}_{i}, \bar{Y}_{i}$, and $\bar{K}_{c}$ satisfying LMIs (31), (34), and (38). Furthermore, the controller gain matrix can be obtained as $K_{c}=\bar{K}_{c} L^{-1}$. If the performance index $\gamma$ is minimised subject to the LMIs (31), (34), and (38), the controller with the optimal performance can be obtained.

\section{Application to Seismic-Excited Building}

In this section, an example is presented to illustrate the applicability and effectiveness of the proposed approach to a seismic-excited building with parameter uncertainties and actuator saturation.

In this example, a three-storey shear building model is considered [30], where the active bracing system (ABS) is installed at the first floor to control the vibration of the structure as shown in Figure 2. It is assumed that all the masses, stiffnesses, and damping coefficients for each floor are identical, and the nominal structural parameters are given as $m_{i}=1,000 \mathrm{~kg}, c_{i}=1.407 \mathrm{kN} \mathrm{s} / \mathrm{m}$, and $k_{i}=980 \mathrm{kN} / \mathrm{m}$, where $i=1,2,3$, respectively. The equation of motion of the three-storey shear building model is obtained similar 
to equation (2), in which

$$
A=\left[\begin{array}{cccccc}
0 & 0 & 0 & 1 & 0 & 0 \\
0 & 0 & 0 & 0 & 1 & 0 \\
0 & 0 & 0 & 0 & 0 & 1 \\
-\left(k_{1}+k_{2}\right) / m_{1} & k_{2} / m_{1} & 0 & -\left(c_{1}+c_{2}\right) / m_{1} & c_{2} / m_{1} & 0 \\
k_{2} / m_{2} & -\left(k_{2}+k_{3}\right) / m_{2} & k_{3} / m_{2} & c_{2} / m_{2} & -\left(c_{2}+c_{3}\right) / m_{2} & c_{3} / m_{2} \\
0 & k_{3} / m_{3} & -k_{3} / m_{3} & 0 & c_{3} / m_{3} & -c_{3} / m_{3}
\end{array}\right],
$$

and

$$
B_{w}=\left[\begin{array}{llllll}
0 & 0 & 0 & -1 & -1 & -1
\end{array}\right]^{T}
$$

The controlled output, $z(t)$, is chosen to be the relative displacement of the first floor, that is,

$$
z(t)=\left[\begin{array}{llllll}
1 & 0 & 0 & 0 & 0 & 0
\end{array}\right] q(t)
$$

Consider the structural parameter uncertainties are applied to the stiffnesses and damping coefficients of all floors and, assume that the uncertainties of stiffnesses and damping coefficients are $40 \%$ of their nominal values, respectively, i.e., the stiffnesses can be varied between $0.6 \times 980$ and $1.4 \times 980 \mathrm{kN} / \mathrm{m}$, and the damping coefficients can be varied between $0.6 \times 1.407$ and $1.4 \times 1.407 \mathrm{kN} \mathrm{s} / \mathrm{m}$. The earthquake excitation considered is the El Centro 1940 earthquake of which peak ground acceleration is scaled to $0.112 \mathrm{~g}$. Assume that the relative displacements and the relative velocities of the three floors are all the measurements available for feedback, that is, state feedback control can be realised. Consider the maximum actuator output force limit $u_{\lim }$ as $1500 \mathrm{~N}$ (about $5 \%$ of building weight), and define $\delta=10, \rho=0.1$ and $h=25 \mathrm{~ms}$. Then, using the approach presented in Section 4.2 and considering parameter uncertainties and saturation limit defined above, we obtain the full state feedback controller gain as

$$
K_{c}=10^{5} \times\left[\begin{array}{llllll}
-2.2156 & -1.8261 & 2.3587 & -0.4203 & -0.2205 & -0.1022
\end{array}\right] .
$$


Now, we firstly check the effect of sampling frequency on the closed-loop (controlled) system performance. As defined above, the controller is designed for $h=25 \mathrm{~ms}$, which means that the sampled controller is able to stabilise the system for any sampling interval less than $25 \mathrm{~ms}$, i.e., sampling frequency larger than $40 \mathrm{~Hz}$. To evaluate the system performance, six evaluation criteria in relation to building responses are used [31]. The first three criteria are based on peak responses of the building and the last three criteria are based on root mean square (RMS) responses of the building. In general, small values of these evaluation criteria are more desirable. The first evaluation criterion is a measure of the normalised maximum floor displacement relative to the ground, given as

$$
J_{1}=\max _{t, i}\left(\frac{\left|x_{i}(t)\right|}{x_{\max }}\right)
$$

where $x_{i}(t)$ is the relative displacement of the $i$ th floor over the entire response, $x_{\text {max }}$ denotes the uncontrolled maximum displacement. The second evaluation criterion is a measure of the reduction in the interstorey drift. The maximum of the normalised interstorey drift is

$$
J_{2}=\max _{t, i}\left(\frac{\left|d_{i}(t)\right|}{d_{\max }}\right)
$$

where $d_{i}(t)$ is the interstorey drift of the above ground floors over the response history, and $d_{\max }$ denotes the peak interstorey drift in the uncontrolled response. The third evaluation criterion is a measure of the peak floor accelerations, given by

$$
J_{3}=\max _{t, i}\left(\frac{\left|\ddot{x}_{a i}(t)\right|}{\ddot{x}_{a \max }}\right)
$$

where $\ddot{x}_{a i}(t)$ is the absolute acceleration of the $i$ th floor, and $\ddot{x}_{a \max }$ is the peak uncontrolled absolute acceleration. The next three criteria are defined for floor displacement $\left(J_{4}\right)$, interstorey $\operatorname{drift}\left(J_{5}\right)$, and floor acceleration $\left(J_{6}\right)$ in their normed-based forms as

$$
\begin{aligned}
& J_{4}=\max _{i}\left(\frac{\left\|x_{i}(t)\right\|}{\left\|x_{\max }\right\|}\right), \\
& J_{5}=\max _{i}\left(\frac{\left\|d_{i}(t)\right\|}{\left\|d_{\max }\right\|}\right), \\
& J_{6}=\max _{i}\left(\frac{\left\|\ddot{x}_{a i}(t)\right\|}{\left\|\ddot{x}_{a \max }\right\|}\right),
\end{aligned}
$$

where $\|\cdot\|$ denotes norm computation, and $\left\|x_{\max }\right\|,\left\|d_{\max }\right\|$, and $\left\|\ddot{x}_{a \max }\right\|$ denote the maximum normed floor displacement, interstorey drift, and floor acceleration corresponding to the uncontrolled structure. 
For the nominal system (no parameter uncertainties), the variations of these six criteria with the change of sampling frequency are shown in Figure 3. It is seen from Figure 3 that the sampling frequency surely affects the system performance as when the sampling frequency is lower than about $40 \mathrm{~Hz}$, six evaluation criteria are relatively larger. On the contrary, when the sampling frequency is higher than about $40 \mathrm{~Hz}$, six evaluation criteria are smaller and do not change significantly with the increase of sampling frequency. Although by the Nyquist sampling theorem, the measured variables need to be sampled at a frequency at least twice the dominant structural frequency to reconstruct the structural response, to achieve a better control performance, the system should be sampled at a relatively higher frequency. In practice, the sampling frequencies of most vibration sensors are in the order of 100-500 Hz, thus the applicability of the designed controller is sufficient.

Next, we will check the robustness of the designed controller. For doing so, we will evaluate the structural responses under the earthquake excitation when system parameters are varied. For brevity, we only consider four-vertex cases where the system stiffnesses and damping coefficients are given as their vertex values, respectively. In the following, Case 1 corresponds to $k_{i}=0.6 \times 980 \mathrm{kN} / \mathrm{m}$ and $c_{i}=0.6 \times 1.407 \mathrm{kN} \mathrm{s} / \mathrm{m}$, Case 2 corresponds to $k_{i}=0.6 \times 980 \mathrm{kN} / \mathrm{m}$ and $c_{i}=1.4 \times 1.407 \mathrm{kN} \mathrm{s} / \mathrm{m}$, Case 3 corresponds to $k_{i}=1.4 \times 980$ $\mathrm{kN} / \mathrm{m}$ and $c_{i}=0.6 \times 1.407 \mathrm{kN} \mathrm{s} / \mathrm{m}$, and Case 4 corresponds to $k_{i}=1.4 \times 980 \mathrm{kN} / \mathrm{m}$ and $c_{i}=1.4 \times 1.407$ $\mathrm{kN} \mathrm{s} / \mathrm{m}$. For comparison, the nominal case, Case 0, is also evaluated. The sampling frequency is used as 50 Hz.

The responses of the open-loop (uncontrolled) system $(u(t)=0)$ and the closed-loop (controlled) system are compared in Figure 4, where only the structural responses of the first floor for Case 0 are shown for clarity. The control force is plotted in Figure 5 which clearly shows the difference between continuous-time signal and sampled signal and the actuation effect on the control signal. For detailed comparison, the maximum open- and closed-loop interstorey drifts, $d_{i \max }, i=1,2,3$, floor absolute accelerations, $\ddot{x}_{a i \max }$, $i=1,2,3$, and control force $u_{\max }$ are summarised in Table 1 , where OL means open-loop and CL means closed-loop. It can be seen from Figure 4 and Table 1 that better responses are obtained for all closed-loop cases no matter there are parameter uncertainties or not. It is validated that the designed controller is robust 


\begin{tabular}{ccccccccccccc}
\hline \hline & \multicolumn{1}{c}{ Case 0} & \multicolumn{2}{c}{ Case 1} & \multicolumn{2}{c}{ Case 2} & \multicolumn{2}{c}{ Case 3} & \multicolumn{2}{c}{ Case 4} \\
\cline { 2 - 9 } Control & OL & CL & OL & CL & OL & CL & OL & CL & OL & CL \\
\hline$u_{\max }(\mathrm{kN})$ & 0 & 1.50 & 0 & 1.50 & 0 & 1.50 & 0 & 1.50 & 0 & 1.50 \\
\hline$d_{1 \max }(\mathrm{cm})$ & 1.35 & 0.37 & 1.67 & 0.72 & 1.53 & 0.69 & 0.82 & 0.23 & 0.71 & 0.23 \\
$d_{2 \max }(\mathrm{cm})$ & 1.03 & 0.39 & 1.36 & 0.80 & 1.24 & 0.76 & 0.66 & 0.26 & 0.57 & 0.26 \\
$d_{3 \max }(\mathrm{cm})$ & 0.60 & 0.22 & 0.80 & 0.52 & 0.68 & 0.49 & 0.40 & 0.15 & 0.34 & 0.14 \\
$\ddot{x}_{a 1 \max }\left(\mathrm{m} / \mathrm{s}^{2}\right)$ & 3.14 & 1.66 & 2.85 & 2.41 & 2.58 & 2.28 & 2.63 & 2.00 & 2.11 & 1.63 \\
$\ddot{x}_{a 2 \max }\left(\mathrm{m} / \mathrm{s}^{2}\right)$ & 4.77 & 2.17 & 3.48 & 2.94 & 3.30 & 2.41 & 4.09 & 2.43 & 3.44 & 2.04 \\
$\ddot{x}_{a 3 \max }\left(\mathrm{m} / \mathrm{s}^{2}\right)$ & 5.88 & 2.17 & 4.69 & 3.05 & 4.01 & 2.87 & 5.46 & 2.10 & 4.67 & 1.95 \\
\hline \hline
\end{tabular}

Table 1: Peak response of the system for scaled El Centro 1940 earthquake record

to parameter uncertainties.

Finally, we change the actuator saturation limit to $u_{\lim }=700 \mathrm{~N}$ (about $2.3 \%$ of building weight) to design a new controller and to evaluate its performance. The newly designed controller is given as

$$
K_{c}=10^{4} \times\left[\begin{array}{llllll}
-5.0092 & -6.8508 & 7.2399 & -1.5720 & -0.8165 & -0.1314
\end{array}\right]
$$

Similarly, the six criteria versus sampling frequency for the nominal system with the newly designed controller are plotted in Figure 6, from which a similar conclusion can be obtained as when the sampling frequency is lower than about $40 \mathrm{~Hz}$, six evaluation criteria are relatively large, and when the sampling frequency is higher than about $40 \mathrm{~Hz}$, six evaluation criteria are smaller. This suggests again that sampling a control system at a relatively higher frequency will yield better control performance.

Under the same earthquake excitation, the structural responses of the first floor and the control force for the nominal system are plotted in Figures 7-8, which show that the closed-loop is stable and the closed-loop performance is a little worse than those obtained with high saturation limit case as shown in Figure 4 . The detailed comparisons for four-vertex cases and the nominal case on the maximum interstorey drifts, $d_{i \max }$, $i=1,2,3$, floor absolute accelerations, $\ddot{x}_{a i \max }, i=1,2,3$, and control force $u_{\max }$ are summarised in Table 2, which confirms again that the closed-loop systems can always achieve good peak response quantities 


\begin{tabular}{ccccccccccccc}
\hline \hline & \multicolumn{2}{c}{ Case 0} & \multicolumn{2}{c}{ Case 1} & \multicolumn{2}{c}{ Case 2} & \multicolumn{2}{c}{ Case 3} & \multicolumn{2}{c}{ Case 4} \\
\cline { 2 - 11 } Control & OL & CL & OL & CL & OL & CL & OL & CL & OL & CL \\
\hline$u_{\max }(\mathrm{kN})$ & 0 & 0.70 & 0 & 0.70 & 0 & 0.70 & 0 & 0.70 & 0 & 0.70 \\
\hline$d_{1 \max }(\mathrm{cm})$ & 1.35 & 0.58 & 1.67 & 1.17 & 1.53 & 1.11 & 0.82 & 0.35 & 0.71 & 0.33 \\
$d_{2 \max }(\mathrm{cm})$ & 1.03 & 0.51 & 1.36 & 1.03 & 1.24 & 0.99 & 0.66 & 0.32 & 0.57 & 0.30 \\
$d_{3 \max }(\mathrm{cm})$ & 0.60 & 0.32 & 0.80 & 0.63 & 0.68 & 0.60 & 0.40 & 0.22 & 0.34 & 0.19 \\
$\ddot{x}_{a 1 \max }\left(\mathrm{m} / \mathrm{s}^{2}\right)$ & 3.14 & 1.73 & 2.85 & 2.16 & 2.58 & 1.97 & 2.63 & 2.03 & 2.11 & 1.68 \\
$\ddot{x}_{a 2 \max }\left(\mathrm{m} / \mathrm{s}^{2}\right)$ & 4.77 & 2.23 & 3.48 & 2.96 & 3.30 & 2.56 & 4.09 & 2.16 & 3.44 & 1.95 \\
$\ddot{x}_{a 3 \max }\left(\mathrm{m} / \mathrm{s}^{2}\right)$ & 5.88 & 3.10 & 4.69 & 3.74 & 4.01 & 3.51 & 5.46 & 3.07 & 4.67 & 2.67 \\
\hline \hline
\end{tabular}

Table 2: Peak response of the system for scaled El Centro 1940 earthquake record

regardless of the parameter uncertainties.

Time delay is one of the important problems in control systems since it may affect the system's stability and performance. When the time delay is mainly from the computation of control laws, there are few cases where the time delay is larger than a sampling interval [32]. In this case, the presented approach can naturally deal with the time delay effect provided that the sum of time delay and sampling interval is less than the designed maximum sampling interval $h$. As shown in Figure 9, when the sampling frequency is $100 \mathrm{~Hz}$ (i.e., sampling interval is $10 \mathrm{~ms}$ ), and the time delay is increased from $20 \%$ to $80 \%$ of the sampling interval, there are no clear degradation on the control performance for the cases where the actuator limit is given as $1500 \mathrm{~N}$ and $700 \mathrm{~N}$, respectively. When the actuator time delays, which are taken by the actuators to build up the required control forces and may be longer than the sampling intervals, are considered in the sampled-data controller design, the methods proposed in [33] to deal with two additive delays could be used. In addition, when actuator dynamics is considered, if it is a linear actuator model as used in [34], this actuator model can be included into the controller design process without introducing much difficulties. If it is a nonlinear actuator model, other techniques such as model-based Takagi-Sugeno fuzzy control strategy would be helpful. Nevertheless, these issues are beyond the focus of the current paper and will not be included. 


\section{Conclusions}

In this paper, a sampled-data controller design approach is presented for structures considering variable sampling rate, actuator saturation, and robust stability with respect to parameter uncertainties. The sampling rate is allowed to be varied in practice when it satisfies the designed minimum sampling frequency requirement. Since parameter uncertainties on stiffness and damping always happen to structures due to changing environmental conditions, and they are difficult to be measured in real time, a robust fixed gain controller is designed in spite of its conservatism compared to the gain-scheduled controller. In addition, for a given saturation limit, a high gain controller can be designed using the presented method to realise better control performance. Simulation example shows that the controllers designed using the presented approach can effectively achieve the attenuation objective when there are parameter uncertainties and actuator saturation constraint.

\section{References}

[1] T. Chen and B. A. Francis. Optimal Sampled-Data Control Systems. Springer-Verlag New York, Inc., Secaucus, NJ, USA, 1995.

[2] P. Shi. Filtering on sampled-data systems with parametric uncertainty. 43(7):1022-1027, 1998.

[3] Z. Wang, B. Huang, and P. Huo. Sampled-data filtering with error covariance assignment. IEEE Transactions on Signal Processing, 49(3):666-670, 2001.

[4] E. Fridman, A. Seuret, and J. P. Richard. Robust sampled-data stabilization of linear systems: An input delay approach. Automatica, 40(8):1441-1446, 2004.

[5] H. Gao and T. Chen. Stabilization of nonlinear systems under variable sampling: A fuzzy control approach. IEEE Transactions on Fuzzy Systems, 15(5):972-983, 2007.

[6] T. T. Soong. State-of-the-art review: Active structural control in civil engineering. Engineering Structures, 10(2):74-84, 1988. 
[7] M. D. Symans and M. C. Constantinou. Semi-active control systems for seismic protection of structures: a state-of-the-art review. Engineering Structures, 21(6):469-487, 1999.

[8] G. Song, V. Sethi, and H.-N. Li. Vibration control of civil structures using piezoceramic smart materials: A review. Engineering Structures, 28(11):1513-1524, 2006.

[9] Y. Lei, A. S. Kiremidjian, K. K. Nair, J. P. Lynch, and K. H. Law. Algorithms for time synchronization of wireless structural monitoring sensors. Earthquake Engineering and Structural Dynamics, 34:555-573, 2005.

[10] C. H. Loh, J. P. Lynch, K. C. Lu, Y. Wang, C. M. Chang, P. Y. Lin, and T. H. Yeh. Experimental verification of a wireless sensing and control system for structural control using MR dampers. Earthquake Engineering and Structural Dynamics, 36:1303-1328, 2007.

[11] S. Park, D. J. Inman, and C.-B. Yun. An outlier analysis of MFC-based impedance sensing data for wireless structural health monitoring of railroad tracks. Engineering Structures, 30(10):2792-2799, 2008.

[12] M. J. Whelan, M. V. Gangone, K. D. Janoyan, and R. Jha. Real-time wireless vibration monitoring for operational modal analysis of an integral abutment highway bridge. Engineering Structures, 31(10):2224-2235, 2009.

[13] J. P. Hespanha, P. Naghshtabrizi, and Y. Xu. A survey of recent results in networked control systems. Proceedings of the IEEE, 95(1):138-162, 2007.

[14] C. C. Lin, K. H. Lu, and L. L. Chung. Optimal discrete-time structural control using direct output feedback. Engineering Structures, 18(6):472-480, 1996.

[15] S. Ankireddi and H. T. Y. Yang. Sampled-data $H_{2}$ optimal output feedback control for civil structures. Earthquake Engineering and Structural Dynamics, 28:921-940, 1999.

[16] T.-W. Ma and H. T. Y. Yang. Sampled-data feedback-feedforward control of structures with time delays. Journal of Structural Engineering, 132(7):1129-1138, 2006. 
[17] S. G. Wang, H. Y. Yen, and P. N. Roschke. Robust control for structural systems with parametric and unstructured uncertainties. Journal of Vibration and Control, 7:753-772, 2001.

[18] W. Gao. Random seismic response analysis of truss structures with uncertain parameters. Engineering Structures, 29(7):1487-1498, 2007.

[19] J. H. Kim and F. Jabbari. Actuator saturation and control design for buildings under seismic excitation. Journal of Engineering Mechanics, 128(4):403-412, 2002.

[20] C. W. Lim, Y. J. Park, and S. J. Moon. Robust saturation controller for linear time-invariant system with structured real parameter uncertainties. Journal of Sound and Vibration, 294:1-14, 2006.

[21] C. W. Lim. Remarks on robust stability of saturation controllers. Journal of Sound and Vibration, 299:363-372, 2007.

[22] C. W. Lim. Multi-input robust saturation controller for uncertain linear time-invariant systems. Journal of Sound and Vibration, 300:1079-1087, 2007.

[23] S. Suresh, S. Narasimhan, S. Nagarajaiah, and N. Sundararajan. Fault-tolerant adaptive control of nonlinear base-isolated buildings using EMRAN. Engineering Structures, 32(8):2477-2487, 2010.

[24] H. Du and N. Zhang. $H_{\infty}$ control for buildings with time delay in control via linear matrix inequalities and genetic algorithms. Engineering Structures, 30(1):81-92, 2008.

[25] J. N. Yang, S. Lin, and F. Jabbari. Performance-based controller for civil engineering structures. In Proceedings of the American Control Conference, pages 1083-1088, Arlington, VA, USA, 2001.

[26] F. Jabbari. Disturbance attenuation of LPV systems with bounded inputs. Dynamics and Control, 11:133-150, 2001.

[27] T. Nguyen and F. Jabbari. Output feedback controllers for disturbance attenuation with actuator amplitude and rate saturation. Automatica, 36:1339-1346, 2000.

[28] T. Nguyen and F. Jabbari. Disturbance attenuation for systems with input saturation: An LMI approach. IEEE Transactions on Automatic Control, 44(4):852-857, 1999. 
[29] Y.-Y. Cao and Z. Lin. Robust stability analysis and fuzzy-scheduling control for nonlinear systems subject to actuator saturation. IEEE Transactions on Fuzzy Systems, 11(1):57-67, 2003.

[30] I. E. Kose, W. E. Schmitendorf, F. Jabbari, and J. N. Yang. $H_{\infty}$ active seismic response control using static output feedback. Journal of Engineering Mechanics, 122(7):651-659, 1996.

[31] Y. Ohtori, R. E. Christenson, B. F. Jr. Spencer, and S. J. Dyke. Benchmark control problems for seismically excited nonlinear buldings. Journal of Engineering Mechanics, 130(4):366-385, 2004.

[32] R. Kondo and K. Furuta. Sampled-data optimal control of continuous systems for quadratic criterion function taking account of delayed control action. International Journal of Control, 41(4):1051-1060, 1985.

[33] H. Gao, T. Chen, and J. Lam. A new delay system approach to network-based control. Automatica, 44:39-52, 2008.

[34] B. Samali, J. N. Yang, and C. T. Yeh. Control of lateral-torsional motion of wind-excited buildings. Journal of Engineering Mechanics, 111(6):777-796, 1985. 


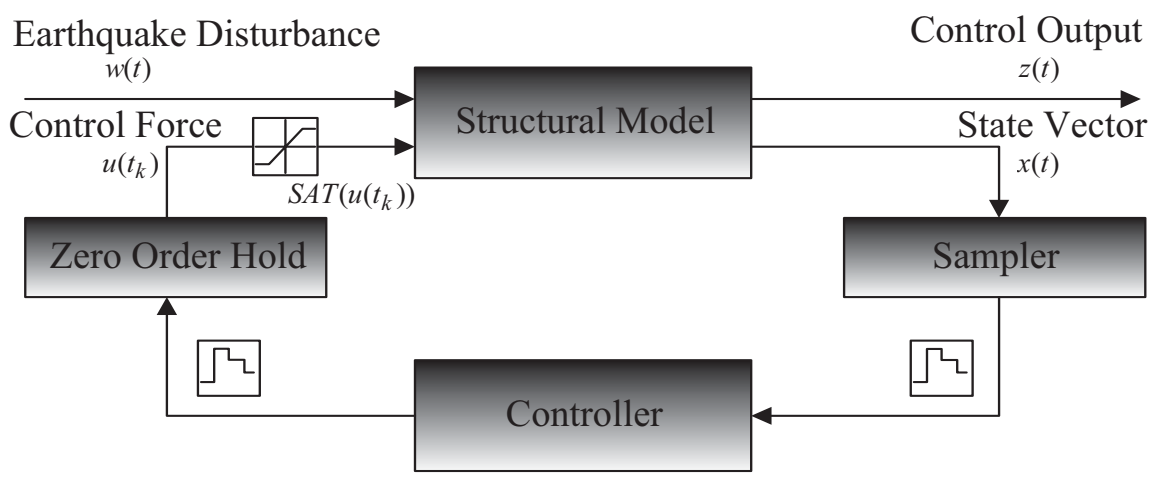

Figure 1: Block diagram for sampled-data control of structure.

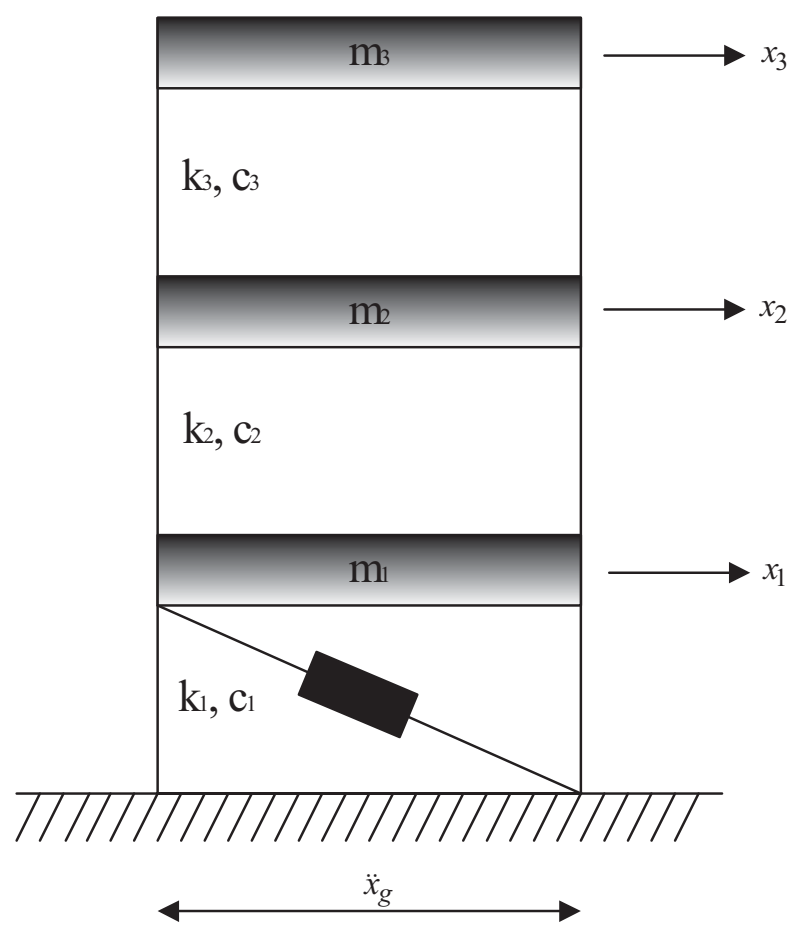

Figure 2: 3 DOF building model with ABS. 


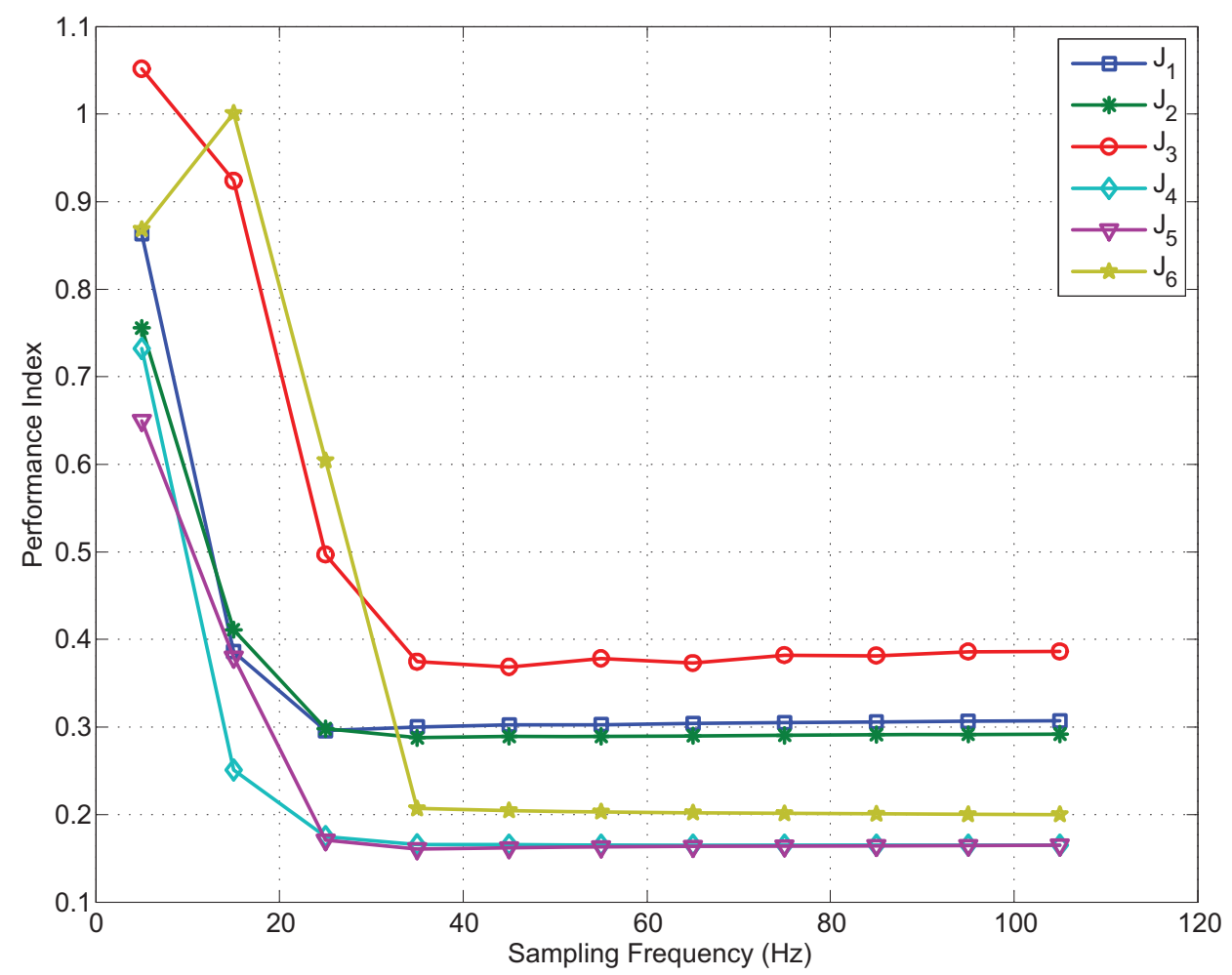

Figure 3: Evaluation criteria values for different sampling frequencies with actuator limit of $1500 \mathrm{~N}$. 

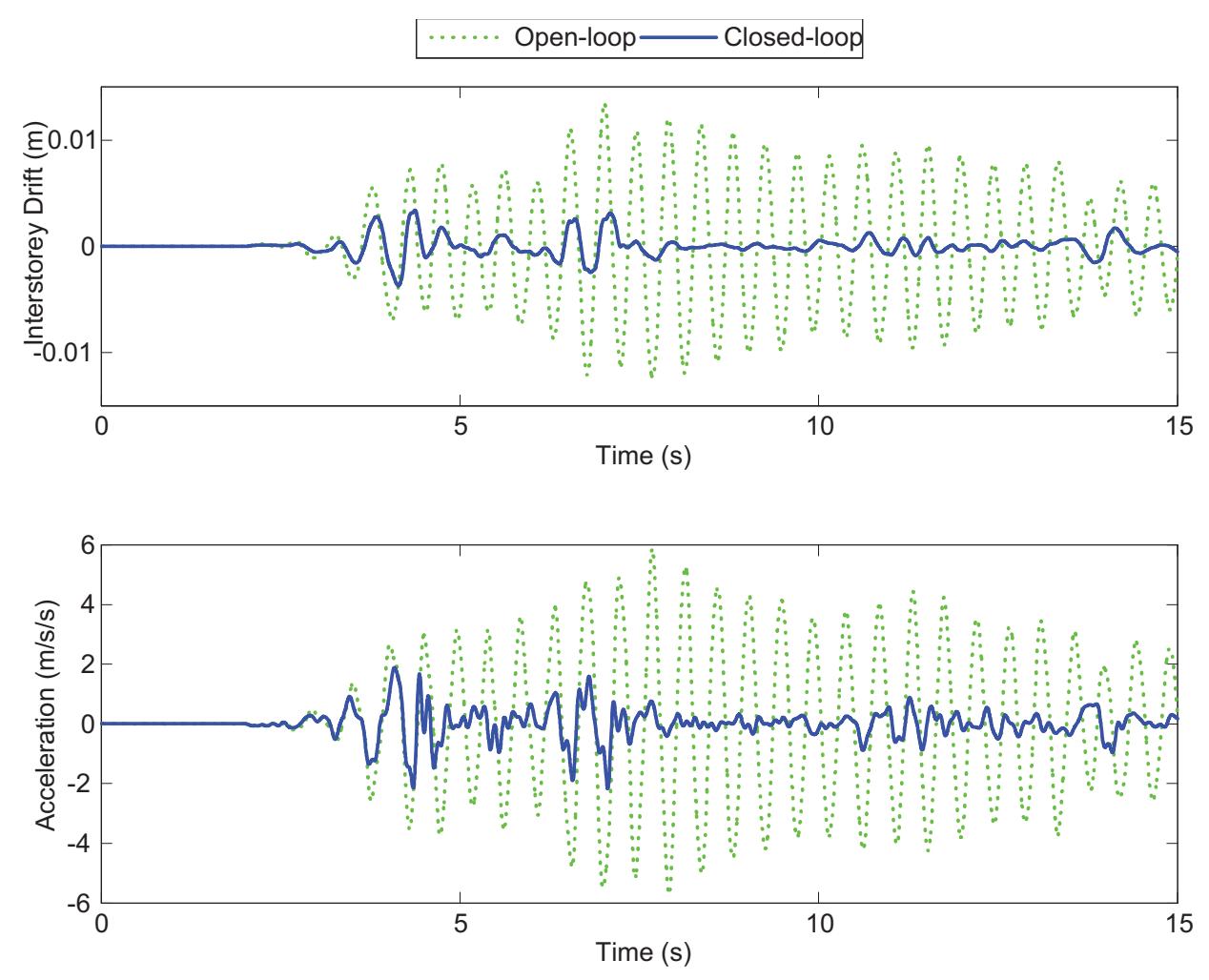

Figure 4: Responses of the first floor for nominal system applying the proposed controller (actuator limit $1500 \mathrm{~N}$ ). 


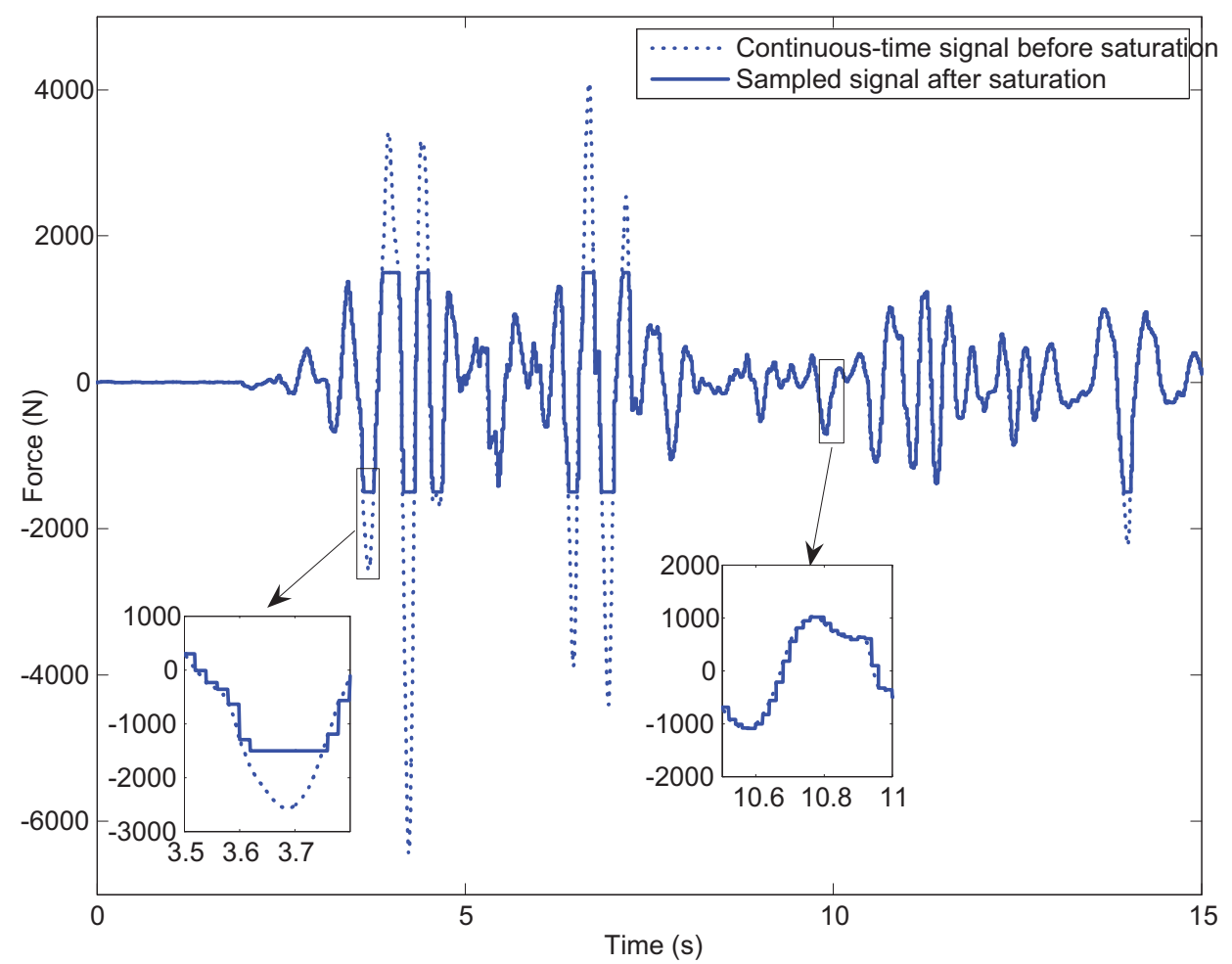

Figure 5: Control force with actuator limit as $1500 \mathrm{~N}$.

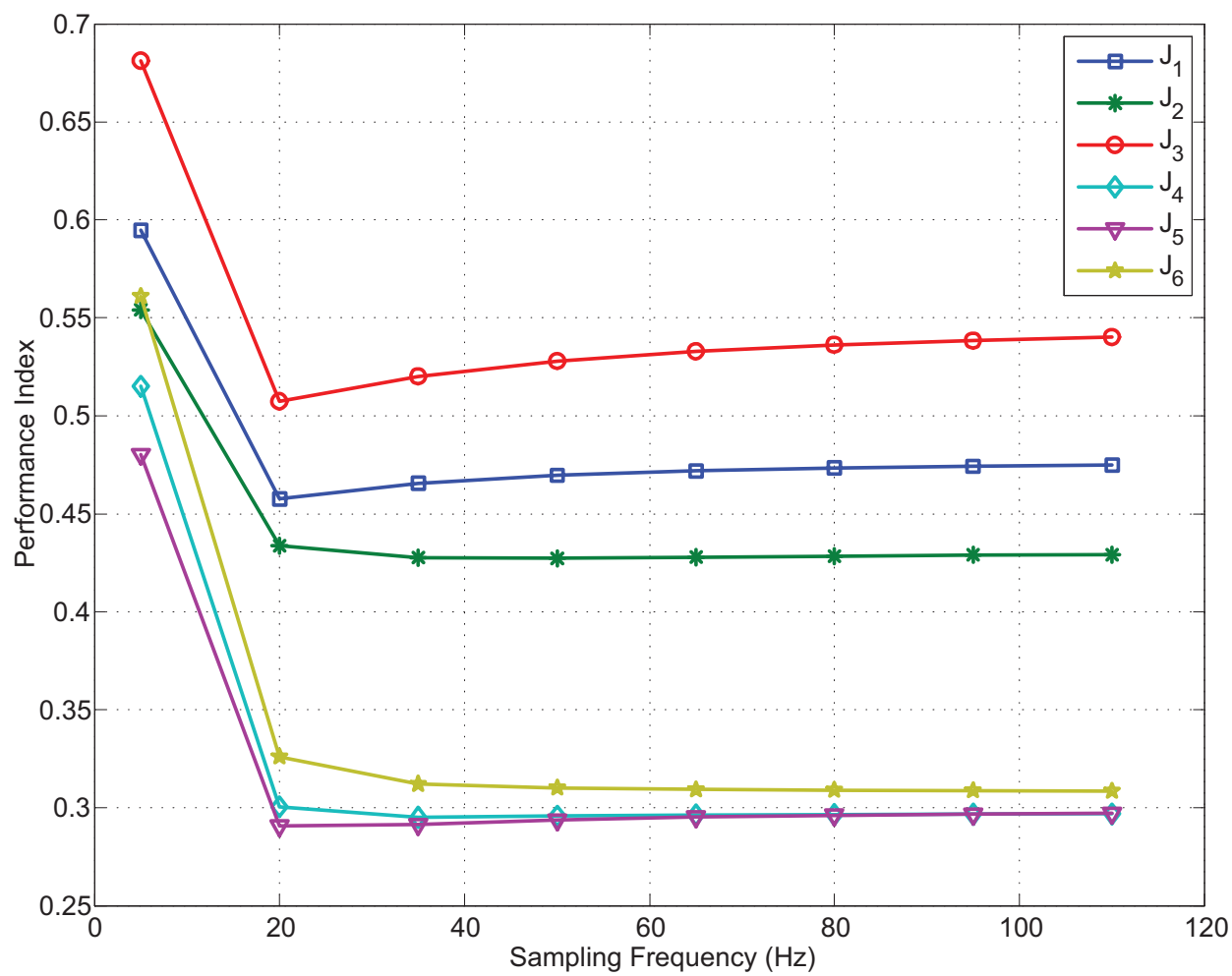

Figure 6: Evaluation criteria values for different sampling frequencies with actuator limit of $700 \mathrm{~N}$. 

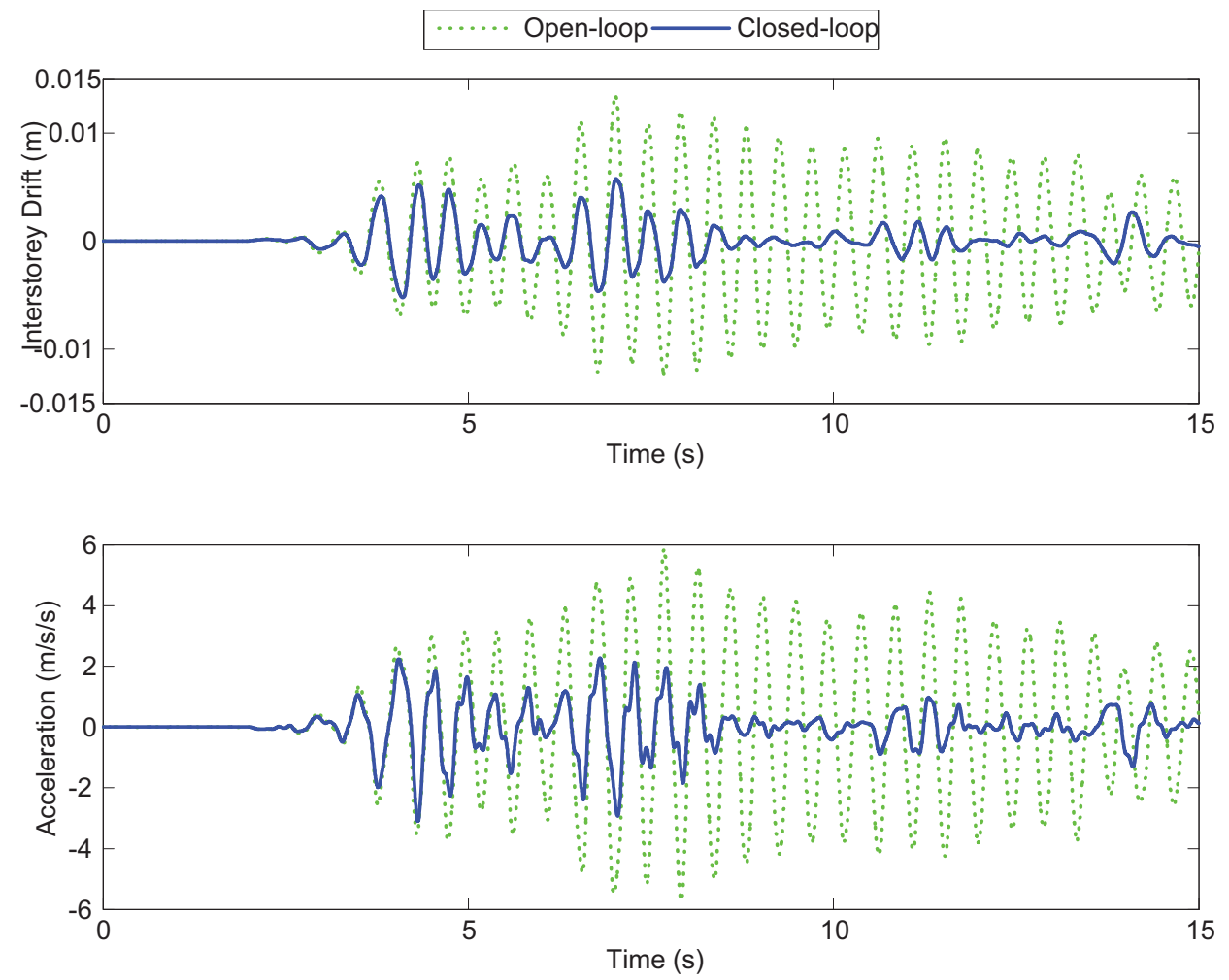

Figure 7: Responses of the first floor for nominal system applying the proposed controller (actuator limit $700 \mathrm{~N})$. 


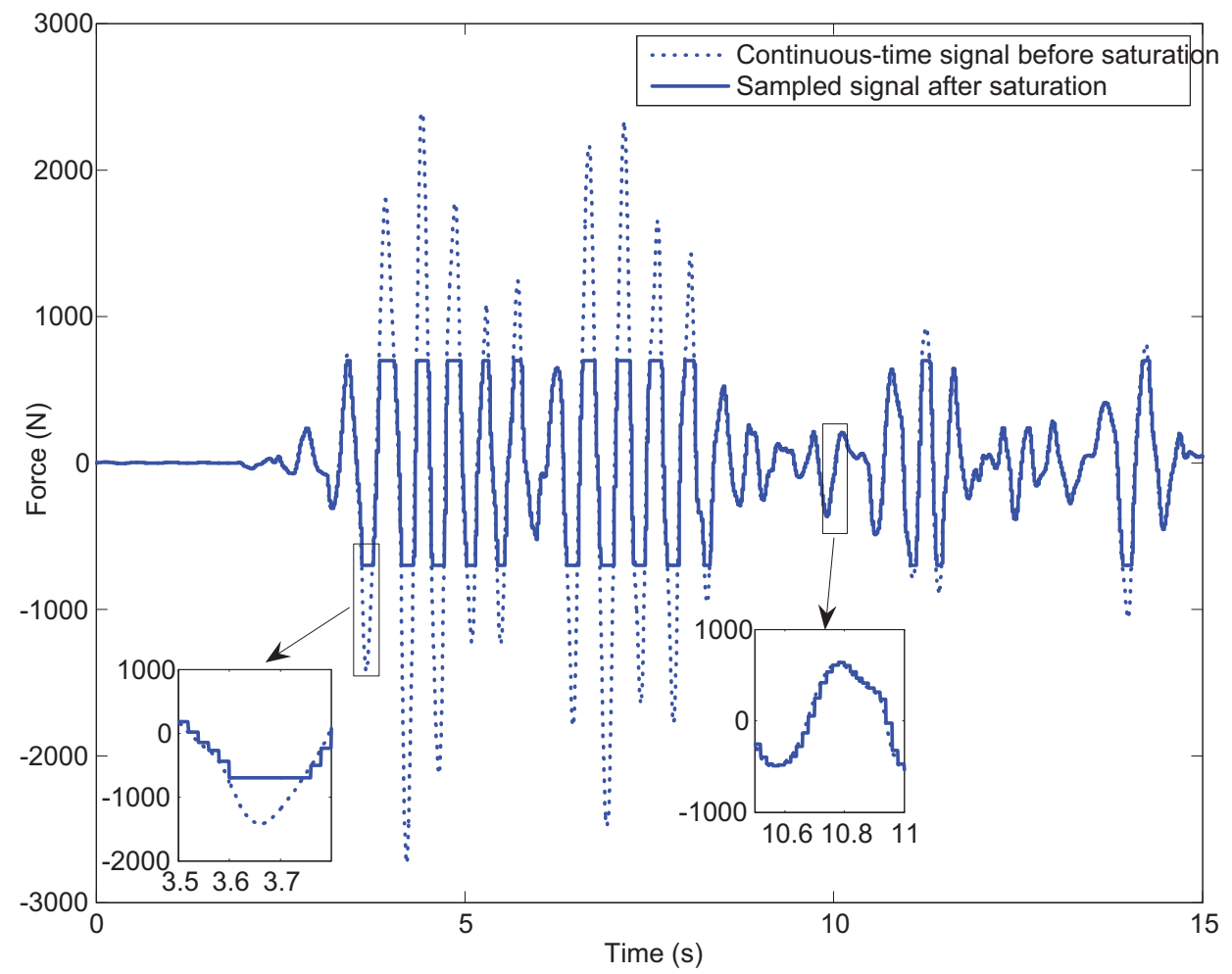

Figure 8: Control force with actuator limit as $700 \mathrm{~N}$.

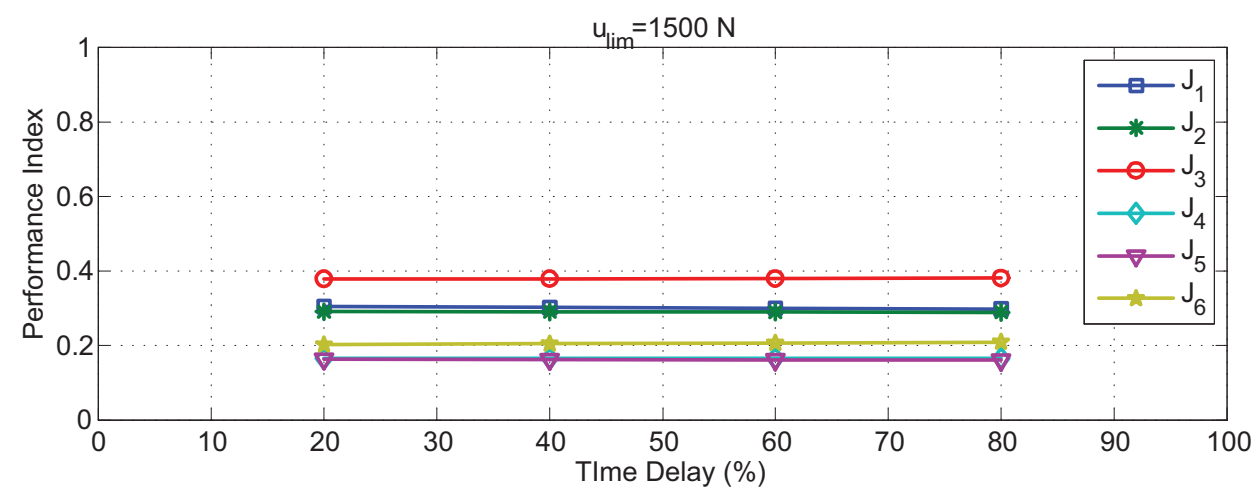

$u_{\lim }=700 \mathrm{~N}$

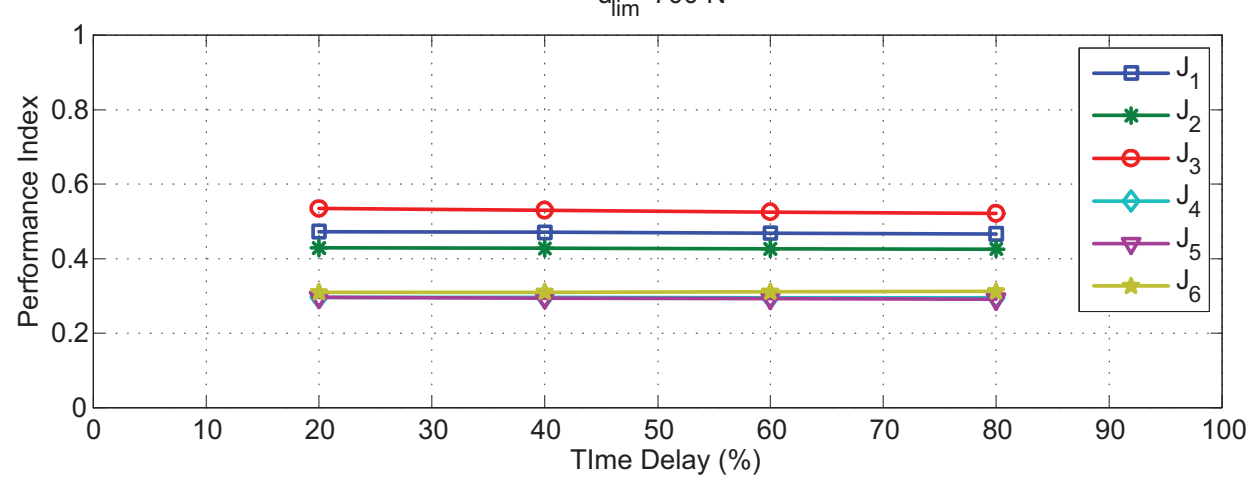

Figure 9: Effects of time delays on control performance. 